\title{
Derivations of First Type of Algebra of Second Class Filiform Leibniz Algebras of Dimension Derivation $(n+2)$
}

\author{
AL-Nashri AL-Hossain Ahmad \\ Department of Mathematics, AL Qunfudha University College, Umm AL Qura University, Makkah, Saudia Arabia
}

Email address:

aanashri@uqu.edu.sa

\section{To cite this article:}

AL-Nashri AL-hossain Ahmad. Derivations of First Type of Algebra of Second Class Filiform Leibniz Algebras of Dimension Derivation (n+2). Pure and Applied Mathematics Journal. Vol. 5, No. 1, 2016, pp. 23-31. doi: 10.11648/j.pamj.20160501.14

\begin{abstract}
This paper describes the derivations of first type of algebra from the second class filiform Leibniz algebras of dimension derivation $(n+2)$. The set of all derivations of an algebra $L$ is denoted by $\operatorname{Der}(L)$ From the description of the derivations, we found the basis of the space $\operatorname{Der}\left(L_{n}(a)\right)$ of the algebra.
\end{abstract}

Keywords: Filiform Leibniz Algebra, Leibniz Algebra, Gradation, Natural Gradation, Derivation

\section{Introduction and Preliminaries}

In mathematics and in particular in the theory of Lie algebra, a Leibniz algebra (which was first introduced by L. Loday in 1993, [5]) is an algebra L over a field K satisfying the following Leibniz identity

$$
[x,[y, z]]=[[x, y], z]-[[x, z], y] .
$$

for any $x, y, z \in L$, where [.,.] denotes the multiplication in L. Leibniz algebra is a generalization of Lie algebra. Onwards, all algebras are assumed to be over the field of complex numbers C. Now, let L be a Leibniz algebra, we put: $L^{1}=L, L^{k+1}=\left[L^{k}, L\right]$ for $k \geq 1$.

Following [7], a Leibniz algebra $L$ is said to be nilpotent if there exists $s \in N$ such that $L^{1} \supset L^{2} \supset \ldots \supset L^{S}=0$. A Leibniz algebra $L$ is said to be filiform if $\operatorname{dim} L^{i}=n-i$, where $n=\operatorname{dim} L$ and $2 \leq i \leq n$, see [1]. Let $\mathrm{d}$ be a K-linear transformation of an algebra $\mathrm{L}, \mathrm{d}$ is called a derivation of $\mathrm{L}$ ([6]) if

$$
d([x, y])=[d(x), y]+[x, d(y)] \text { for all } x, y \in L .
$$

The set of all derivations of an algebra $L$ is denoted by $\operatorname{Der}(L)$. We also, denote by Leibniz the set of all $(\mathrm{n}+1)$-dimensional filiform Leibniz algebras. We now look at the following theorem from [3] which splits the set of fixed dimension filiform Liebniz algebras into three disjoint subsets. However we just take the result of this theorem regarding only FLeib $b_{n+1}$.

Theorem 1.1 Any $(n+1)$-dimensional complex filiform Leibniz algebra $L$ admits a basis $e_{0}, e_{1}, \ldots, e_{n}$ called adapted, such that the table of multiplication of $L$ has the following forms, where non defined products are zero:

$$
\text { SLeib }_{n+1}=\left\{\begin{array}{l}
{\left[e_{0}, e_{0}\right]=e_{2},} \\
{\left[e_{i}, e_{0}\right]=e_{i+1}, 2 \leq i \leq n-1} \\
{\left[e_{0}, e_{1}\right]=\beta_{3} e_{3}+\beta_{4} e_{4}+\ldots+\beta_{n-2} e_{n-1}+\beta_{n-1} e_{n},} \\
{\left[e_{1}, e_{1}\right]=\gamma e_{n-1}} \\
{\left[e_{j}, e_{1}\right]=\beta_{3} e_{j+2}+\beta_{4} e_{j+3}+\ldots+\beta_{n+1-j} e_{n}, 2 \leq j \leq n-2,}
\end{array}\right.
$$

for $\beta_{3}, \beta_{4}, \ldots, \beta_{n}, \beta \in C$.

Lemma 1.1 [6] Let $d \in \operatorname{Der}\left(L_{n}\right)$. In this case $d=d_{0}+d_{1}+d_{2}+\ldots+d_{n-1}$ where $d_{k} \in \operatorname{End}\left(L_{n}\right)$ and $d_{k}\left(L_{i}\right) \subseteq L_{i+k}$ for $1 \leq i \leq n$. 
The purpose of this paper is to study the low dimension of algebras in order to get the basis of the space $\operatorname{Der}\left(L_{n}(a)\right)$. We attempt to find the basis of the derivation for this algebra and the relationship between the algebra and its derivations, by studying the table of this algebra from dimension 5 to 15 .

\section{Algebra $L_{n}(a)$ of the Second Class Filiform Leibniz Algebras}

$$
L_{n}(a)=\left\{\begin{array}{l}
{\left[e_{0}, e_{0}\right]=e_{2},} \\
{\left[e_{i}, e_{0}\right]=e_{i+1}, \quad 2 \leq i \leq n-1 .} \\
{\left[e_{0}, e_{1}\right]=e_{n} .}
\end{array}\right.
$$

We observe the derivations of this type of algebra in low dimension in the following table:

Table 1. Derivations of $\operatorname{Der}\left(L_{n}(a)\right)$ with low dimension.

\begin{tabular}{|c|c|c|c|}
\hline dimension & equation(dim Der) & dim Der & No. of equations \\
\hline 5 & $\begin{array}{c}d_{1}\left(e_{0}\right)=e_{0}, d_{1}\left(e_{1}\right)=3 e_{1} \\
d_{1}\left(e_{i}\right)=i e_{i}, 2 \leq i \leq 4 \\
d_{2}\left(e_{0}\right)=e_{1}, d_{2}\left(e_{2}\right)=e_{4} \\
d_{3}\left(e_{0}\right)=e_{2}, d_{3}\left(e_{i}\right)=e_{i+1}, 2 \leq i \leq 3 \\
d_{4}\left(e_{0}\right)=e_{3}, d_{4}\left(e_{2}\right)=e_{4} \\
d_{5}\left(e_{0}\right)=e_{4}, d_{6}\left(e_{1}\right)=e_{4}\end{array}$ & 6 & 14 \\
\hline 6 & $\begin{array}{c}d_{1}\left(e_{0}\right)=e_{0}, d_{1}\left(e_{1}\right)=4 e_{1} \\
d_{1}\left(e_{i}\right)=i e_{i}, 2 \leq i \leq 5 \\
d_{2}\left(e_{0}\right)=e_{1}, d_{2}\left(e_{2}\right)=e_{5} d_{3}\left(e_{0}\right)=e_{2} \\
d_{3}\left(e_{i}\right)=e_{i+1}, 2 \leq i \leq 4 \\
d_{4}\left(e_{0}\right)=e_{3} \\
d_{4}\left(e_{i}\right)=e_{i+1}, 2 \leq i \leq 3 \\
d_{5}\left(e_{0}\right)=e_{4}, d_{5}\left(e_{2}\right)=e_{5} \\
d_{6}\left(e_{0}\right)=e_{5}, d_{7}\left(e_{1}\right)=e_{5}\end{array}$ & 7 & 19 \\
\hline 7 & $\begin{array}{c}d_{1}\left(e_{0}\right)=e_{0}, d_{1}\left(e_{1}\right)=5 e_{1}, \\
d_{1}\left(e_{i}\right)=i e_{i}, 2 \leq i \leq 6 \quad d_{2}\left(e_{0}\right)=e_{1}, \\
d_{2}\left(e_{2}\right)=e_{6} d_{3}\left(e_{0}\right)=e_{2}, d_{3}\left(e_{i}\right)=e_{i+1}, 2 \leq i \leq 5 \\
d_{4}\left(e_{0}\right)=e_{3}, d_{4}\left(e_{i}\right)=e_{i+1}, 2 \leq i \leq 4 \\
d_{5}\left(e_{0}\right)=e_{4}, d_{5}\left(e_{i}\right)=e_{i+1}, 2 \leq i \leq 3 \\
d_{6}\left(e_{0}\right)=e_{4}, d_{6}\left(e_{2}\right)=e_{6}, \\
d_{7}\left(e_{0}\right)=e_{6}, d_{8}\left(e_{1}\right)=e_{6} .\end{array}$ & 8 & 25 \\
\hline 8 & $\begin{array}{c}d_{1}\left(e_{0}\right)=e_{0}, d_{1}\left(e_{1}\right)=6 e_{1}, \\
d_{1}\left(e_{i}\right)=i e_{i}, 2 \leq i \leq 7 \quad d_{2}\left(e_{0}\right)=e_{1}, \\
d_{2}\left(e_{2}\right)=e_{7} d_{3}\left(e_{0}\right)=e_{2}, d_{3}\left(e_{i}\right)=e_{i+1}, 2 \leq i \leq 6 \\
d_{4}\left(e_{0}\right)=e_{3}, d_{4}\left(e_{i}\right)=e_{i+1}, 2 \leq i \leq 5 \\
d_{5}\left(e_{0}\right)=e_{4}, d_{5}\left(e_{i}\right)=e_{i+1}, 2 \leq i \leq 4 \\
d_{6}\left(e_{0}\right)=e_{5}, d_{6}\left(e_{i}\right)=e_{i+1}, 2 \leq i \leq 3 \\
d_{7}\left(e_{0}\right)=e_{6}, d_{7}\left(e_{2}\right)=e_{7}, \\
d_{8}\left(e_{0}\right)=e_{7}, d_{9}\left(e_{1}\right)=e_{7} .\end{array}$ & 9 & 32 \\
\hline 9 & $\begin{array}{c}d_{1}\left(e_{0}\right)=e_{0}, d_{1}\left(e_{1}\right)=7 e_{1}, \\
d_{1}\left(e_{i}\right)=i e_{i}, 2 \leq i \leq 8 \quad d_{2}\left(e_{0}\right)=e_{1}, d_{2}\left(e_{2}\right)=e_{8} \\
d_{3}\left(e_{0}\right)=e_{2}, d_{3}\left(e_{i}\right)=e_{i+1}, 2 \leq i \leq 7 \\
d_{4}\left(e_{0}\right)=e_{3}, d_{4}\left(e_{i}\right)=e_{i+1}, 2 \leq i \leq 6 \\
d_{5}\left(e_{0}\right)=e_{4}, d_{5}\left(e_{i}\right)=e_{i+1}, 2 \leq i \leq 5 \\
d_{6}\left(e_{0}\right)=e_{5}, d_{6}\left(e_{i}\right)=e_{i+1}, 2 \leq i \leq 4 \\
d_{7}\left(e_{0}\right)=e_{6}, d_{6}\left(e_{i}\right)=e_{i+1}, 2 \leq i \leq 3 \\
d_{8}\left(e_{0}\right)=e_{7}, d_{8}\left(e_{2}\right)=e_{8} \\
d_{9}\left(e_{0}\right)=e_{8}, d_{10}\left(e_{1}\right)=e_{8} .\end{array}$ & 10 & 40 \\
\hline
\end{tabular}




\begin{tabular}{|c|c|c|c|}
\hline dimension & equation(dim Der) & dim Der & No. of equations \\
\hline 11 & $\begin{array}{c}d_{1}\left(e_{0}\right)=e_{0}, d_{1}\left(e_{1}\right)=9 e_{1}, \\
d_{1}\left(e_{i}\right)=i e_{i}, 2 \leq i \leq 10 \quad d_{2}\left(e_{0}\right)=e_{1}, \\
d_{2}\left(e_{2}\right)=e_{10} d_{3}\left(e_{0}\right)=e_{2}, d_{3}\left(e_{i}\right)=e_{i+1}, 2 \leq i \leq 9 \\
d_{4}\left(e_{0}\right)=e_{3}, d_{4}\left(e_{i}\right)=e_{i+1}, 2 \leq i \leq 8 \\
d_{5}\left(e_{0}\right)=e_{4}, d_{5}\left(e_{i}\right)=e_{i+1}, 2 \leq i \leq 7 \\
d_{6}\left(e_{0}\right)=e_{5}, d_{6}\left(e_{i}\right)=e_{i+1}, 2 \leq i \leq 6 \\
d_{7}\left(e_{0}\right)=e_{6}, d_{6}\left(e_{i}\right)=e_{i+1}, 2 \leq i \leq 5 \\
d_{8}\left(e_{0}\right)=e_{7}, d_{8}\left(e_{i}\right)=e_{i+1}, 2 \leq i \leq 4 \\
d_{9}\left(e_{0}\right)=e_{8}, d_{9}\left(e_{i}\right)=e_{i+1}, 2 \leq i \leq 3 \\
d_{10}\left(e_{0}\right)=e_{9}, d_{10}\left(e_{2}\right)=e_{10} \\
d_{11}\left(e_{0}\right)=e_{10}, d_{12}\left(e_{1}\right)=e_{10}\end{array}$ & 12 & 59 \\
\hline 12 & $\begin{array}{c}d_{1}\left(e_{0}\right)=e_{0}, d_{1}\left(e_{1}\right)=10 e_{1}, \\
d_{1}\left(e_{i}\right)=i e_{i}, 2 \leq i \leq 11 d_{2}\left(e_{0}\right)=e_{1}, \\
d_{2}\left(e_{2}\right)=e_{11} d_{3}\left(e_{0}\right)=e_{2}, d_{3}\left(e_{i}\right)=e_{i+1}, 2 \leq i \leq 10 \\
d_{4}\left(e_{0}\right)=e_{3}, d_{4}\left(e_{i}\right)=e_{i+1}, 2 \leq i \leq 9 \\
d_{5}\left(e_{0}\right)=e_{4}, d_{5}\left(e_{i}\right)=e_{i+1}, 2 \leq i \leq 8 \\
d_{6}\left(e_{0}\right)=e_{5}, d_{6}\left(e_{i}\right)=e_{i+1}, 2 \leq i \leq 7 \\
d_{7}\left(e_{0}\right)=e_{6}, d_{6}\left(e_{i}\right)=e_{i+1}, 2 \leq i \leq 6 \\
d_{8}\left(e_{0}\right)=e_{7}, d_{8}\left(e_{i}\right)=e_{i+1}, 2 \leq i \leq 5 \\
d_{9}\left(e_{0}\right)=e_{8}, d_{9}\left(e_{i}\right)=e_{i+1}, 2 \leq i \leq 4 \\
d_{10}\left(e_{0}\right)=e_{9}, d_{10}\left(e_{i}\right)=e_{i+1}, 2 \leq i \leq 3 \\
d_{11}\left(e_{0}\right)=e_{10}, d_{11}\left(e_{2}\right)=e_{11}, \\
d_{12}\left(e_{0}\right)=e_{11}, d_{13}\left(e_{1}\right)=e_{11} .\end{array}$ & 13 & 70 \\
\hline 13 & $\begin{array}{c}d_{1}\left(e_{0}\right)=e_{0}, d_{1}\left(e_{1}\right)=11 e_{1}, \\
d_{1}\left(e_{i}\right)=i e_{i}, 2 \leq i \leq 12 \quad d_{2}\left(e_{0}\right)=e_{1}, \\
d_{2}\left(e_{2}\right)=e_{12} \\
d_{3}\left(e_{0}\right)=e_{2}, d_{3}\left(e_{i}\right)=e_{i+1}, 2 \leq i \leq 11 \\
d_{4}\left(e_{0}\right)=e_{3}, d_{4}\left(e_{i}\right)=e_{i+1}, 2 \leq i \leq 10 \\
d_{5}\left(e_{0}\right)=e_{4}, d_{5}\left(e_{i}\right)=e_{i+1}, 2 \leq i \leq 9 \\
d_{6}\left(e_{0}\right)=e_{5}, d_{6}\left(e_{i}\right)=e_{i+1}, 2 \leq i \leq 8 \\
d_{7}\left(e_{0}\right)=e_{6}, d_{6}\left(e_{i}\right)=e_{i+1}, 2 \leq i \leq 7 \\
d_{8}\left(e_{0}\right)=e_{7}, d_{8}\left(e_{i}\right)=e_{i+1}, 2 \leq i \leq 6 \\
d_{9}\left(e_{0}\right)=e_{8}, d_{9}\left(e_{i}\right)=e_{i+1}, 2 \leq i \leq 5 \\
d_{10}\left(e_{0}\right)=e_{9}, d_{10}\left(e_{i}\right)=e_{i+1}, 2 \leq i \leq 4 \\
d_{11}\left(e_{0}\right)=e_{10}, d_{11}\left(e_{i}\right)=e_{i+1}, 2 \leq i \leq 3 \\
d_{12}\left(e_{0}\right)=e_{11}, d_{12}\left(e_{2}\right)=e_{12} \\
d_{13}\left(e_{0}\right)=e_{12}, d_{14}\left(e_{1}\right)=e_{12}\end{array}$ & 14 & 82 \\
\hline 14 & $\begin{array}{c}d_{1}\left(e_{0}\right)=e_{0}, d_{1}\left(e_{1}\right)=12 e_{1}, \\
d_{1}\left(e_{i}\right)=i e_{i}, 2 \leq i \leq 13 d_{2}\left(e_{0}\right)=e_{1}, \\
d_{2}\left(e_{2}\right)=e_{13} d_{3}\left(e_{0}\right)=e_{2}, d_{3}\left(e_{i}\right)=e_{i+1}, 2 \leq i \leq 12 \\
d_{4}\left(e_{0}\right)=e_{3}, d_{4}\left(e_{i}\right)=e_{i+1}, 2 \leq i \leq 11 \\
d_{5}\left(e_{0}\right)=e_{4}, d_{5}\left(e_{i}\right)=e_{i+1}, 2 \leq i \leq 10 \\
d_{6}\left(e_{0}\right)=e_{5}, d_{6}\left(e_{i}\right)=e_{i+1}, 2 \leq i \leq 9 \\
d_{7}\left(e_{0}\right)=e_{6}, d_{6}\left(e_{i}\right)=e_{i+1}, 2 \leq i \leq 8 \\
d_{8}\left(e_{0}\right)=e_{7}, d_{8}\left(e_{i}\right)=e_{i+1}, 2 \leq i \leq 7 \\
d_{9}\left(e_{0}\right)=e_{8}, d_{9}\left(e_{i}\right)=e_{i+1}, 2 \leq i \leq 6 \\
d_{10}\left(e_{0}\right)=e_{9}, d_{10}\left(e_{i}\right)=e_{i+1}, 2 \leq i \leq 5 \\
d_{11}\left(e_{0}\right)=e_{10}, d_{11}\left(e_{i}\right)=e_{i+1}, 2 \leq i \leq 4 \\
d_{12}\left(e_{0}\right)=e_{11}, d_{12}\left(e_{i}\right)=e_{i+1}, 2 \leq i \leq 3 \\
d_{13}\left(e_{0}\right)=e_{12}, d_{13}\left(e_{2}\right)=e_{13}, \\
d_{14}\left(e_{0}\right)=e_{13}, d_{15}\left(e_{1}\right)=e_{13} .\end{array}$ & 15 & 95 \\
\hline
\end{tabular}




\begin{tabular}{|c|c|c|c|}
\hline dimension & equation(dim Der) & $\operatorname{dim}$ Der & No. of equations \\
\hline 15 & $\begin{array}{c}d_{1}\left(e_{0}\right)=e_{0}, d_{1}\left(e_{1}\right)=13 e_{1}, \\
d_{1}\left(e_{i}\right)=i e_{i}, 2 \leq i \leq 14 d_{2}\left(e_{0}\right)=e_{1}, \\
d_{2}\left(e_{2}\right)=e_{14} d_{3}\left(e_{0}\right)=e_{2}, d_{3}\left(e_{i}\right)=e_{i+1}, 2 \leq i \leq 13 \\
d_{4}\left(e_{0}\right)=e_{3}, d_{4}\left(e_{i}\right)=e_{i+1}, 2 \leq i \leq 12 \\
d_{5}\left(e_{0}\right)=e_{4}, d_{5}\left(e_{i}\right)=e_{i+1}, 2 \leq i \leq 11 \\
d_{6}\left(e_{0}\right)=e_{5}, d_{6}\left(e_{i}\right)=e_{i+1}, 2 \leq i \leq 10 \\
d_{7}\left(e_{0}\right)=e_{6}, d_{6}\left(e_{i}\right)=e_{i+1}, 2 \leq i \leq 9 \\
d_{8}\left(e_{0}\right)=e_{7}, d_{8}\left(e_{i}\right)=e_{i+1}, 2 \leq i \leq 8 \\
d_{9}\left(e_{0}\right)=e_{8}, d_{9}\left(e_{i}\right)=e_{i+1}, 2 \leq i \leq 7 \\
d_{10}\left(e_{0}\right)=e_{9}, d_{10}\left(e_{i}\right)=e_{i+1}, 2 \leq i \leq 6 \\
d_{11}\left(e_{0}\right)=e_{10}, d_{11}\left(e_{i}\right)=e_{i+1}, 2 \leq i \leq 5 \\
d_{12}\left(e_{0}\right)=e_{11}, d_{12}\left(e_{i}\right)=e_{i+1}, 2 \leq i \leq 4 \\
d_{13}\left(e_{0}\right)=e_{12}, d_{13}\left(e_{i}\right)=e_{i+1}, 2 \leq i \leq 3 \\
d_{14}\left(e_{0}\right)=e_{13}, d_{14}\left(e_{2}\right)=e_{3}, \\
d_{15}\left(e_{0}\right)=e_{14}, d_{16}\left(e_{1}\right)=e_{14} .\end{array}$ & 16 & 109 \\
\hline
\end{tabular}

Notes:

From Table 1, we obtain the main result of this paper: We are able to find a basis of the space $\operatorname{Der}\left(L_{n}(a)\right)$ and this is given in the proposition 2.1. Therefore we can find the dim Der of this algebra for any dimension by using this rule: $\operatorname{dim} \operatorname{Der}\left(L_{n}\right)=\operatorname{dim}\left(L_{n}\right)=n+2$, for $n \geq 3$. Finally, we observe that the number of equations that can be calculated for any dimension obtained from derivations the following rule: = $\frac{n(n+1)}{2}+4$.

Let $d \in \operatorname{Der}\left(L_{n}(a)\right)$. By using Lemma 2.1, we have $d=d_{0}+d_{1}+d_{2}+d_{3}+d_{4}+\ldots+d_{n-2}+d_{n-1}$.

In Lemma 3.1, we give definition for $d_{0} \in \operatorname{Der}\left(L_{n}(a)\right)$.

In Lemma 3.2 , we give definition for $d_{k} \in \operatorname{Der}\left(L_{n}(a)\right), 1 \leq k \leq(n-2)$.

In Lemma 3.3 , we give definition for $d_{n-1} \in \operatorname{Der}\left(L_{n}(a)\right)$.

We are going to find the basis of algebra $L_{n}(a)$.

For this purpose, we present the following Lemma (2.1, 2.2 and 2.3 ).

Lemma 2.1 Let $t_{1}=\lambda_{0} e_{0}+(n-1) e_{1}+\sum_{i=2}^{n} \lambda_{i} e_{i}$. Then, we have

$t_{1}\left(e_{0}\right)=e_{0}, \quad t_{1}\left(e_{1}\right)=(n-1) e_{1}$ and $t_{1}\left(e_{i}\right)=i e_{i}, 2 \leq i \leq n-1$.

Proof. Consider $d_{0} \in \operatorname{Der}\left(L_{n}(a)\right)$ which is defined by

$$
d_{0}\left(e_{i}\right)=\left\{\begin{array}{l}
\alpha_{0} e_{0}, i=0 \\
\alpha_{1}(n-1) e_{1} \quad i=1, \\
\alpha_{i} i e_{i}, \quad 2 \leq i \leq n,
\end{array}\right.
$$

where $\alpha_{i}, 0 \leq i \leq n$, are scalars. Consider the family of derivations

$$
d_{0}\left(\left[e_{i}, e_{j}\right]\right)=\left[d_{0}\left(e_{i}\right), e_{j}\right]+\left[e_{i}, d_{0}\left(e_{j}\right)\right] .
$$

We now look at the problem case by case. In each case, we repeatedly use algebra $L_{n}(a)$ and (1).

Case 1: if $i=0$ and $j=0$, then

$$
d_{0}\left(\left[e_{0}, e_{0}\right]\right)=\left[d_{0}\left(e_{0}\right), e_{0}\right]+\left[e_{0}, d_{0}\left(e_{0}\right)\right]
$$

and so,

$$
d_{0}\left(e_{2}\right)=\left[\alpha_{0} e_{0}, e_{0}\right]+\left[e_{0}, \alpha_{0} e_{0}\right]
$$

which implies

$$
2 \alpha_{2} e_{2}=\alpha_{0} e_{2}+\alpha_{0} e_{2}
$$

and thus

$$
\alpha_{2}=\alpha_{0}
$$

Case 2: if $2 \leq i \leq n, j=0$ then

$$
d_{0}\left(\left[\sum_{i=2}^{n} e_{i}, e_{0}\right]\right)=\left[d_{0}\left(\sum_{i=2}^{n} e_{i}\right), e_{0}\right]+\left[\sum_{i=2}^{n} e_{i}, d_{0}\left(e_{0}\right)\right]
$$

so that,

$$
d_{0}\left(\sum_{i=2}^{n-1} e_{i+1}\right)=\left[\sum_{i=2}^{n} \alpha_{i} e_{i}, e_{0}\right]+\left[\sum_{i=2}^{n} e_{i}, \alpha_{0} e_{0}\right]
$$

which implies

$$
\begin{gathered}
\sum_{i=2}^{n-1} \alpha_{i+1}(i+1) e_{i+1}=\sum_{i=2}^{n-1} i \alpha_{i} e_{i+1}+\sum_{i=2}^{n-1} \alpha_{0} e_{i+1} \\
\sum_{i=2}^{n-1} \alpha_{i+1}(i+1) e_{i+1}=\sum_{i=2}^{n-1}\left(i \alpha_{i}+\alpha_{0}\right) e_{i+1}
\end{gathered}
$$

and so,

$$
(i+1) \alpha_{i+1}=i \alpha_{i}+\alpha_{0}, \quad 2 \leq i \leq n-1
$$

If $\mathrm{i}=2$ in (3), then $3 \alpha_{3}=2 \alpha_{2}+\alpha_{0}$. By (2) we obtain 


$$
\alpha_{3}=\alpha_{2}
$$

If $\mathrm{i}=3$ in (3) then $4 \alpha_{4}=3 \alpha_{3}+\alpha_{0}$. By (2) and (4), we obtain

$$
\alpha_{4}=\alpha_{3}
$$

Similarly, if $\mathrm{i}=\mathrm{n}-1$ in (3) then

$$
\text { (n) } \alpha_{n}=(n-1) \alpha_{n-1}+\alpha_{0} \text {. }
$$

But $\alpha_{0}=\alpha_{n-1}$. Thus,

$$
\alpha_{n}=\alpha_{n-1}
$$

From (2), (4), (5) and (6) we obtain

$$
\alpha_{0}=\alpha_{2}=\alpha_{3}=\alpha_{4}=\cdots=\alpha_{n}
$$

Thus, using (1) and (7), we get

$$
\begin{aligned}
& d_{0}\left(\sum_{i=0}^{n} \lambda_{i} e_{i}\right)=d_{0}\left(\lambda_{0} e_{0}\right)+d_{0}\left(\lambda_{1} e_{1}\right)+d_{0}\left(\lambda_{2} e_{2}\right)+\sum_{i=3}^{n} d_{0}\left(\lambda_{i} e_{i}\right) \\
= & \lambda_{0}\left(\alpha_{0} e_{0}\right)+\lambda_{1}\left(\alpha_{0}(n-1) e_{1}\right)+2 \lambda_{2}\left(\alpha_{2} e_{2}\right)+\sum_{i=3}^{n} i \lambda_{i}\left(\alpha_{i} e_{i}\right) \\
= & \alpha_{0}\left[\lambda_{0} e_{0}+\lambda_{1}(n-1) e_{1}+2 \lambda_{2} e_{2}+\sum_{i=3}^{n} i \lambda_{i} e_{i}\right] \\
= & \alpha_{0} t_{1} .
\end{aligned}
$$

From this we obtain

$$
t_{1}\left(e_{0}\right)=e_{1}, \quad t_{1}\left(e_{1}\right)=(n-1) e_{1} \text { and } t_{1}\left(e_{i}\right)=i e_{i}, 2 \leq i \leq n-1
$$

Lemma 2.1 gives some vectors of the basis. In the next we will complete this basis.

Lemma 2.2 Consider $d_{k} \in \operatorname{Der}\left(L_{n}(a)\right), \quad 1 \leq k \leq n-2$ such that $d_{k}$ is defined as

$$
d_{k}\left(e_{i}\right)=\left\{\begin{array}{rr}
\lambda_{0} e_{k}, i=0, & 1 \leq k \leq n \\
\lambda_{i} e_{k+i-1}, & 2 \leq i \leq n-1 \\
& 2 \leq k \leq n-1
\end{array}\right.
$$

where $\lambda_{0}$ and $\lambda_{i}, 1 \leq i \leq n-k$ are constants.

Then $d_{k}\left(e_{0}\right)=e_{k}$ and $d_{k}\left(e_{i}\right)=e_{i+k-1}$ for $2 \leq i \leq n$ and $2 \leq k \leq n-1$.

Proof. Consider the family of derivations

$$
d_{k}\left(\left[e_{i}, e_{j}\right]\right)=\left[d_{k}\left(e_{i}\right), e_{j}\right]+\left[e_{i}, d_{k}\left(e_{j}\right)\right]
$$

Similarly, we calculate case by case. In which we repeatedly $L_{n}(a)$ and $(9)$.

Case 1: if $i=0, j=0$, then

$$
\sum_{k=1}^{n-1} d_{k}\left(\left[e_{0}, e_{0}\right]\right)=\left[\sum_{k=1}^{n-1} d_{k}\left(e_{0}\right), e_{0}\right]+\left[e_{0}, \sum_{k=1}^{n-1} d_{k}\left(e_{0}\right)\right]
$$

Then

$$
\sum_{k=1}^{n-1} d_{k}\left(e_{2}\right)=\left[\sum_{k=1}^{n} \lambda_{0} e_{k}, e_{0}\right]+\left[e_{0}, \sum_{k=1}^{n} \lambda_{0} e_{k}\right]
$$

which implies

$$
\sum_{k=1}^{n-1} \lambda_{2} e_{k+1}=\sum_{k=1}^{n-1} \lambda_{0} e_{k+1}+\left[e_{0}, \lambda_{0} e_{1}\right]
$$$$
\sum_{k=1}^{n-1} \lambda_{2} e_{k+1}=\sum_{k=1}^{n-2} \lambda_{0} e_{k+1}+\lambda_{0} e_{n}
$$

$$
\sum_{k=1}^{n-1} \lambda_{2} e_{k+1}=\sum_{k=1}^{n-2} \lambda_{0} e_{k+1}+\lambda_{0} e_{n}
$$

$$
\sum_{k=1}^{n-1} \lambda_{2} e_{k+1}=\sum_{k=1}^{n-1} \lambda_{0} e_{k+1}
$$

to obtain

$$
\lambda_{2}=\lambda_{0}
$$

Case 2: if $1 \leq i \leq n-1, j=0$ and $2 \leq k \leq n-1$, then

$$
\sum_{k=2}^{n-1} \sum_{i=1}^{n-1} d_{k}\left(\left[e_{i}, e_{0}\right]\right)=\left[\sum_{k=2}^{n-1} \sum_{i=1}^{n-1} d_{k}\left(e_{i}\right), e_{0}\right]+\left[\sum_{i=1}^{n-1} e_{i}, \sum_{k=2}^{n-1} d_{k}\left(e_{0}\right)\right]
$$

$$
\sum_{k=1}^{n-1} \sum_{i=2}^{n-1} d_{k}\left(e_{i+1}\right)=\left[\sum_{k=1}^{n-1} \sum_{i=1}^{n-1} \lambda_{i} e_{k+i-1}, e_{0}\right]+\left[\sum_{i=1}^{n-1} e_{i}, \sum_{k=1}^{n-1} d_{k}\left(e_{0}\right)\right]
$$

$$
\begin{gathered}
\sum_{k=1}^{n-1} \sum_{i=2}^{n-1} \lambda_{i+1}\left(e_{i+k}\right)=\left[\sum_{k=1}^{n-1} \sum_{i=1}^{n-1} \lambda_{i} e_{k+i-1}, e_{0}\right]+\left[\sum_{i=1}^{n-1} e_{i}, \sum_{k=1}^{n-1} \lambda_{0}\left(e_{k}\right)\right] \\
\sum_{k=1}^{n-1} \sum_{i=2}^{n-1} \lambda_{i+1} e_{i+k}=\sum_{k=1}^{n-1} \sum_{i=1}^{n-1} \lambda_{i} e_{k+i}
\end{gathered}
$$

Thus,

$$
\lambda_{i+1}=\lambda_{i}
$$

From (11), if $i=2$, then $\lambda_{3}=\lambda_{2}$. Also, if $i=3$ then $\lambda_{4}=\lambda_{3}$. Similarly, if $i=n-1$, then $\lambda_{n}=\lambda_{n-1}$. From (10) and (11) this implies

$$
\lambda_{0}=\lambda_{2}=\lambda_{3}=\ldots=\lambda_{n-1}=\lambda_{n}
$$

and hence, by (9) and (7), we get 


$$
\begin{aligned}
d_{k}\left(\sum_{i=0}^{n} \alpha_{i} e_{i}\right) & =d_{k}\left(\alpha_{0} e_{0}\right)+d_{k}\left(\alpha_{1} e_{1}\right)+\sum_{i=2}^{n} d_{k}\left(\alpha_{i} e_{i}\right) \\
& =\alpha_{0}\left(\lambda_{0} e_{k}\right)+\sum_{i=2}^{n} \lambda_{i}\left(\alpha_{i} e_{k+i-1}\right) \\
& =\alpha_{0}\left(\lambda_{0} e_{k}+\sum_{i=2}^{n-k} \lambda_{i} e_{k+i}\right)
\end{aligned}
$$

Thus,

$$
d_{k}\left(e_{0}\right)=e_{k} \text { and } d_{k}\left(e_{i}\right)=e_{k+i}, 2 \leq i \leq n-k .
$$

This conclude the proof.

Lemma 2.2 gives a second part of the basis of Algebra. Up to now, we obtained two parts of the basis. This imcomplete basis will be completed by other vectors will be given Lemma 2.3

Lemma 2.3 Let $t_{2}=\lambda_{0} e_{n}$. Then $t_{2}\left(e_{0}\right)=e_{n}$.

Proof. Consider $d_{n-1} \in \operatorname{Der}\left(L_{n}(a)\right)$ where $d_{n-1}$ is defined by

$$
d_{n-1}\left(e_{0}\right)=\pi_{0} e_{n},
$$

where $\pi_{0}$ is a constant. Consider the family of derivations

$$
d_{n-1}\left(\left[e_{i}, e_{j}\right]\right)=\left[d_{n-1}\left(e_{i}\right), e_{j}\right]+\left[e_{i}, d_{n-1}\left(e_{j}\right)\right] .
$$

Case 1: if $i=0, j=0$ then

$$
d_{n-1}\left(\left[e_{0}, e_{0}\right]\right)=\left[d_{n-1}\left(e_{0}\right), e_{0}\right]+\left[e_{0}, d_{n-1}\left(e_{0}\right)\right]
$$

by $L_{n}(a)$ and (13), thus

$$
d_{n-1}\left(e_{2}\right)=\left[\pi_{0} e_{n}, e_{0}\right]+\left[e_{0}, \pi_{0} e_{n}\right] .
$$

If $\eta_{0} \neq 0$ then

$$
0=0+0 .
$$

Hence, using (14), we get

$$
\begin{aligned}
d_{n-1}\left(\sum_{i=0}^{n} \lambda_{i} e_{i}\right) & =d_{n-1}\left(\lambda_{0} e_{0}\right)+d_{n-1}\left(\lambda_{1} e_{1}\right)+\sum_{i=2}^{n} d_{n-1}\left(\lambda_{i} e_{i}\right) \\
& =\lambda_{0}\left(\pi_{0} e_{n}\right) \\
& =\pi_{0}\left(\lambda_{0} e_{n}\right) \\
& =\left(\pi_{0} t_{2}\right)
\end{aligned}
$$

and we obtain

$$
t_{2}\left(e_{0}\right)=e_{n} .
$$

This conclude the proof of Lemma 2.3.

In Lemma 2.4 Fullfilment to conclude our study we will now turn to show that the set of vectors given in Lemma 2.1, Lemma 2.2 and Lemma2.3 form a basis of Algebra.

For that, we will show that the set vectors are linearly independent.

Lemma 2.4. The mappings $t_{1}, t_{2}$ and $d_{k}$ for $1 \leq k \leq(n-2)$ are linearly independent.

Proof. Consider that

$$
\alpha_{1} t_{1}\left(e_{i}\right)+\alpha_{2} t_{2}\left(e_{i}\right)+\sum_{k=1}^{n-2} \beta_{k} d_{k}\left(e_{i}\right)=0
$$

where $e_{i} \in L_{n}(a), i=0,1,2,3, \ldots, n-1$. We will show that $\alpha_{1}=\alpha_{2}=\beta_{1}=\beta_{2}=\ldots=\beta_{k}=0$ for $1 \leq k \leq n-2$.

$$
\begin{gathered}
\sum_{i=0}^{n-1}\left[\alpha_{1} t_{1}\left(e_{i}\right)+\alpha_{2} t_{2}\left(e_{i}\right)+\sum_{k=1}^{n-2} \beta_{k} d_{k}\left(e_{i}\right)\right] \\
=\sum_{i=0}^{n-1}\left[\alpha_{1} t_{1}\left(e_{i}\right)+\alpha_{2} t_{2}\left(e_{i}\right)+\beta_{1} d_{1}\left(e_{i}\right)+\beta_{2} d_{2}\left(e_{i}\right)+\ldots\right. \\
\left.+\beta_{n-3} d_{n-3}\left(e_{i}\right)+\beta_{n-2} d_{n-2}\left(e_{i}\right)\right] \\
+\left[\alpha_{1} t_{1}\left(e_{1}\right)+\alpha_{2} t_{2}\left(e_{1}\right)+\alpha_{3} t_{3}\left(e_{1}\right)+\beta_{1} d_{1}\left(e_{1}\right)+\beta_{2} d_{2}\left(e_{1}\right)+\ldots+\beta_{n-3} d_{n-3}\left(e_{1}\right)+\beta_{n-2} d_{n-2}\left(e_{1}\right)\right] \\
+\left[\alpha_{1} t_{1}\left(e_{2}\right)+\alpha_{2} t_{2}\left(e_{2}\right)+\alpha_{3} t_{3}\left(e_{2}\right)+\beta_{1} d_{1}\left(e_{2}\right)+\beta_{2} d_{2}\left(e_{2}\right)+\ldots+\beta_{n-3} d_{n-3}\left(e_{2}\right)+\beta_{n-2} d_{n-2}\left(e_{2}\right)\right] \\
+\left[\alpha_{1} t_{1}\left(e_{3}\right)+\alpha_{2} t_{2}\left(e_{3}\right)+\alpha_{3} t_{3}\left(e_{3}\right)+\beta_{1} d_{1}\left(e_{3}\right)+\beta_{2} d_{2}\left(e_{3}\right)+\ldots+\beta_{n-3} d_{n-3}\left(e_{3}\right)+\beta_{n-2} d_{n-2}\left(e_{3}\right)\right] \\
+\left[\alpha_{1} t_{1}\left(e_{4}\right)+\alpha_{2} t_{2}\left(e_{4}\right)+\alpha_{3} t_{3}\left(e_{4}\right)+\beta_{1} d_{1}\left(e_{4}\right)+\beta_{2} d_{2}\left(e_{4}\right)+\ldots+\beta_{n-3} d_{n-3}\left(e_{4}\right)+\beta_{n-2} d_{n-2}\left(e_{4}\right)\right] \\
+\ldots \\
+\ldots
\end{gathered}
$$




$$
\begin{gathered}
+\left[\alpha_{1} t_{1}\left(e_{n-2}\right)+\alpha_{2} t_{2}\left(e_{n-2}\right)+\alpha_{3} t_{3}\left(e_{n-2}\right)+\beta_{1} d_{1}\left(e_{n-2}\right)+\beta_{2} d_{2}\left(e_{n-2}\right)+\ldots+\beta_{n-3} d_{n-3}\left(e_{n-2}\right)\right. \\
\left.+\beta_{n-2} d_{n-2}\left(e_{n-2}\right)\right]+\left[\alpha_{1} t_{1}\left(e_{n-1}\right)+\alpha_{2} t_{2}\left(e_{n-1}\right)+\alpha_{3} t_{3}\left(e_{n-1}\right)+\beta_{1} d_{1}\left(e_{n-1}\right)+\beta_{2} d_{2}\left(e_{n-1}\right)+\ldots\right. \\
\left.+\beta_{n-3} d_{n-3}\left(e_{n-1}\right)+\beta_{n-2} d_{n-2}\left(e_{n-1}\right)\right] \\
=0 .
\end{gathered}
$$

This implies

$$
\begin{gathered}
\left(\alpha_{1} e_{0}+\beta_{1} e_{2}+\beta_{1} e_{1}+\beta_{2} e_{2}+\beta_{3} e_{3}+\beta_{4} e_{4}+\ldots+\beta_{n-3} e_{n-3}+\beta_{n-2} e_{n-2}+\beta_{n-1} e_{n-1}+\alpha_{2} e_{n}\right) \\
+\left(\alpha_{1}(n-1) e_{1}\right) \\
+\left(2 \alpha_{1} e_{2}+\beta_{2} e_{3}+\beta_{3} e_{4}+\beta_{4} e_{5}+\beta_{5} e_{6}+\ldots+\beta_{n-3} e_{n-2}+\beta_{n-2} e_{n-1}+\beta_{n-1} e_{n}\right) \\
+\left(3 \alpha_{1} e 3+\beta_{2} e_{4}+\beta_{3} e_{5}+\beta_{4} e_{6}+\beta_{5} e_{7}+\ldots+\beta_{n-3} e_{n-1}+\beta_{n-2} e_{n}\right) \\
+\left(4 \alpha_{1} e 4+\beta_{2} e_{5}+\beta_{3} e_{6}+\beta_{4} e_{7}+\beta_{5} e_{8}+\ldots+\beta_{n-4} e_{n-1}+\beta_{n-3} e_{n}\right) \\
+\ldots \\
+\ldots \\
+\left((n-2) \alpha_{1} e_{n-2}+\beta_{2} e_{n-1}+\beta_{3} e_{n}\right) \\
+\left((n-1) \alpha_{1} e_{n-1}+\beta_{2} e_{n}\right) \\
=0
\end{gathered}
$$

We thus have

$$
\begin{gathered}
\alpha_{1} e_{0}+\left(\alpha_{1}(n-1)+\beta_{1}\right) e_{2}+\left(2 \alpha_{1}+\beta_{2}\right) e_{2}+\left(3 \alpha_{1}+\beta_{2}+\beta_{3}\right) e_{3}+\left(4 \alpha_{1}+\beta_{2}+\beta_{3}+\beta_{4}\right) e_{4} \\
+\left(5 \alpha_{1}+\beta_{2}+\beta_{3}+\beta_{4}+\beta_{5}\right) e_{5}+\left(6 \alpha_{1}+\beta_{2}+\beta_{3}+\beta_{4}+\beta_{5}+\beta_{6}\right) e_{6} \\
+\left(7 \alpha_{1}+\beta_{2}+\beta_{3}+\beta_{4}+\beta_{5}+\beta_{6}+\beta_{7}\right) e_{7} \\
+\ldots \\
+\ldots \\
+\left((n-2) \alpha_{1}+\beta_{2}+\beta_{3}+\beta_{4}+\ldots+\beta_{n-2}\right) e_{n-2} \\
+\left((n-1) \alpha_{1}+\beta_{2}+\beta_{3}+\beta_{4}+\ldots+\beta_{n-1}\right) e_{n-1} \\
+\left(\alpha_{2}+\beta_{2}+\beta_{3}+\beta_{4}+\ldots+\beta_{n-1}\right) e_{n} \\
=0 .
\end{gathered}
$$

Here we have these following results:

1. $\alpha_{1} e_{1}=0$ which implies $\alpha_{1}=0$.

2. $\left(\alpha_{1}(n-1)+\beta_{1}\right) e_{2}=0$ which implies $\alpha_{1}(n-1)+\beta_{1}=0$, but since $\alpha_{1}=0$ then $\beta_{1}=0$.

3. $\left(3 \alpha_{1}+\beta_{2}+\beta_{3}\right) e_{3}=0$ which implies $3 \alpha_{1}+\beta_{2}+\beta_{3}=0$, but since $\alpha_{1}=\beta_{2}=0$ then $\beta_{3}=0$.

4. $\left(4 \alpha_{1}+\beta_{2}+\beta_{3}+\beta_{4}\right) e_{4}=0$ which implies $4 \alpha_{1}+\beta_{2}+\beta_{3}+\beta_{4}=0$ but since $\alpha_{1}=\beta_{2}=\beta_{3}=0$, then $\beta_{4}=0$.

5. $\left(5 \alpha_{1}+\beta_{2}+\beta_{3}+\beta_{4}+\beta_{5}\right) e_{5}=0$ which implies $5 \alpha_{1}+\beta_{2}+\beta_{3}+\beta_{4}+\beta_{5}=0$, but since $\alpha_{1}=\beta_{2}=\beta_{3}=\beta_{4}=0$ then $\beta_{4}=0$.

6. Similarly, $\left(\alpha_{2}+\beta_{2}+\beta_{3}+\beta_{4}+\ldots+\beta_{n-1}\right) e_{n}=0 \quad$ which implies $\quad \alpha_{2}+\beta_{2}+\beta_{3}+\beta_{4}+\ldots+\beta_{n-1}=0 \quad$ but $\quad$ since $\alpha_{2}=\beta_{2}=\beta_{3}=\beta_{4}=\beta_{5}=\beta_{6}=\ldots=\beta_{n-1}=0$. Then, we get $\alpha_{2}=0$. 
From of the above we will obtain

$$
\alpha_{1}=\alpha_{2}=\beta_{1}=\beta_{2}=\beta_{3}=\beta_{4}=\beta_{5}=\ldots=\beta_{n-1}=0 .
$$

This conclude our proof and the mappings are linearly independent.

Lemma $2.5 \quad$ The linear mappings
$d_{0}, d_{k}, d_{n-1} \in \operatorname{Der}\left(L_{n}(a)\right), 1 \leq k \leq n-2$ defined by (1), (9) and (14) are linearly composition.

Proof. Let

$$
x=\eta_{0} e_{0}+\eta_{1} e_{1}+\eta_{2} e_{2}+\eta_{3} e_{3}+\eta_{4} e_{4}+\ldots+\eta_{n-1} e_{n-1} .
$$

First we observe that by (1), (9) and (14),

$$
d_{k}(x)=\left\{\begin{array}{l}
2 \eta_{0}\left(\alpha_{1} e_{1}\right)+\eta_{1}\left(\alpha_{0}(n-1)\right) e_{1}+\sum_{2}^{n} \alpha_{i}\left(\eta_{i} i e_{i}\right) \\
+\sum_{k=1}^{n} \eta_{0} \lambda_{0} e_{k}+\sum_{k=2 i=2}^{n-1} \sum_{i=1}^{n-1} \lambda_{i} \eta_{i} e_{k+i-1}+\eta_{0} \pi_{0} e_{n} .
\end{array}\right.
$$

Hence by using (7) and (12),

$$
\begin{gathered}
d(x)=\alpha_{0}\left[\eta_{0}\left(e_{1}\right)+\eta_{1}(n-1) e_{1}+\sum_{2}^{n} \eta_{i} i e_{i}\right] \quad t_{1}(x . y)=\beta_{0}\left(2 \alpha_{0} e_{2}+3 \alpha_{2} e_{3}+4 \alpha_{3} e_{4}+\right. \\
\text { Thus, } \\
t_{1}(x)=\alpha_{0} e_{0}+\alpha_{1}(n-1) e_{1}+2 \alpha_{2} e_{2}+3 \alpha_{3} e_{3}+4 \alpha_{4} e_{4}+\ldots+\alpha_{n-1}(n-1) e_{n-1}
\end{gathered}
$$

Hence

$$
t_{1}(x) \cdot y=\beta_{0}\left[\alpha_{0} e_{2}+2 \alpha_{2} e_{3}+3 \alpha_{3} e_{4}+4 \alpha_{4} e_{5}+\ldots+(n-1)\left(\alpha_{n-1}+\alpha_{1}\right) e_{n}\right]
$$

and thus,

$$
t_{1}(y)=\beta_{0} e_{0}+\beta_{1}(n-1) e_{1}+2 \beta_{2} e_{2}+3 \beta_{3} e_{3}+4 \beta_{4} e_{4}+\ldots+\beta_{n-1}(n-1) e_{n-1}
$$

Therefore,

$x . t_{1}(y)=\beta_{0}\left[\alpha_{0} e_{2}+\alpha_{2} e_{3}+\alpha_{3} e_{4}+\ldots+\left(\alpha_{n-1}+\alpha_{1}\right) e_{n}\right]$

By adding (20) to (21) we will obtain (19). This implies $t_{1}$ is a derivation.

We now show that $t_{2}$ is also a derivation.

$$
t_{2}(x)=\alpha_{0} e_{n}
$$

and

$$
t_{2}(y)=\beta_{0} e_{n}
$$

From easy calculation we have $t_{2}(x) . y=0, x . t_{2}(y)=0$ and $t_{2}(x \cdot y)=0$ and thus $t_{2}$ is a derivation.

Now, since

$$
d_{k}(x)=\sum_{k=1}^{n-1}\left[\alpha_{0} d_{k}\left(e_{0}\right)+\alpha_{1} d_{k}\left(e_{1}\right)+\alpha_{2} d_{k}\left(e_{2}\right)+\ldots+\alpha_{n-1} d_{k}\left(e_{n-1}\right)\right]
$$

thus,

$$
\sum_{k=1}^{n-1} d_{k}(x)=\sum_{k=1}^{n-1}\left[\alpha_{0} e_{k}+\sum_{i=2}^{n-k} \alpha_{i} e_{k+i-1}\right]
$$

then,

$$
\left[d_{k}(x) \cdot y\right]=\beta_{0}\left[\sum _ { k = 1 } ^ { n - 1 } \left(e_{k+1}+\sum_{k=1}^{n-1}\left(\alpha_{2} e_{k+2}+\alpha_{3} e_{k+3}\right.\right.\right.
$$

$$
\left.\left.\left.+\ldots+\alpha_{n-k} e_{n-k}\right)\right)\right] \text {. }
$$

In addition,

$$
\sum_{k=1}^{n-1} d_{k}(y)=\sum_{k=1}^{n-1}\left[\alpha_{0} e_{k}+\sum_{i=2}^{n-k} \alpha_{i} e_{k+i-1}\right]
$$

and we have 


$$
\left[x, \sum_{k=1}^{n-1} d_{k}(y)\right]=0
$$

Also,

$$
\sum_{k=1}^{n-1} d_{k}(x . y)=\sum_{k=1}^{n-1} \beta_{0}\left(\alpha_{0} e_{k+1}+\alpha_{2} e_{k+2}+\alpha_{3} e_{k+3}+\ldots+\alpha_{n-k} e_{n-1} .\right)
$$

By adding (22) to (23) we will get (24), thus $d_{k}$ is a derivation.

This complete the proof of the derivation.

We recall that the $t_{1}, t_{2}$, and $d_{k}$ for $1 \leq k \leq n-2$ are defined in Lemma $2.4, \ldots$, Lemma 2.6 .

We will conclude our discussion with the following Proposition.

$$
\text { Proposition } 2.1 \text { Let } L_{n}(a) \text { be }
$$$$
e_{0} e_{0}=e_{2}, e_{i} e_{0}=e_{i+1}, 2 \leq i \leq n-1 \text { and } e_{1} e_{0}=e_{n} \text {. Then }
$$

$t_{1}, t_{2}$ and $d_{k}$ for $1 \leq k \leq n-2$ form a basis of the space $\operatorname{Der}\left(L_{n}(a)\right)$.

Proof.: The proof follows from Lemma 3.4 to Lemma 3.6

\section{Conclusion}

Finally, the purpose of this manuscript is fourfold:

1. This algebra $L_{n}(a)$, is nilpotent, but it is not characteristically nilpotent.

2. This algebra $L_{n}(a)$ work with basis derivations from five dimension and above.

3. We can find number derivations of this algebra on any dimension by this rule:

$$
\operatorname{dim} \operatorname{Der}\left(L_{n}(a)\right)=n+2 .
$$

4. We can determine the number of equations from the result of derivations by this rule, i.e, the number of equations is equal to $\frac{n(n+1)}{2}+4$.

\section{References}

[1] Albeverio, S., Omirov, B. A., Rakhimov, I. S., (2006), Classification of 4-dimensional nilpotent complex Leibniz algebras, Extracta Math., 3(2006), 197-210.

[2] Dixmier. J. and Lister. W. G., Derivations of nilpotent Lie algebras, Proc. Amer. Math. Soc. 8(1957), 155-158.

[3] M. Goze AND Khakimdjanov, Nilpotent Lie algebras, printed in the netherlands, (1996), $336 \mathrm{p}$.

[4] Jacobson. N., A note on automorphisms and derivations of Lie algebras, Proc. Amer. Math. Soc. 6(1955), 281-283.

[5] Loday. J. -L., Une version non commutative dés algébras de Lie: les algébras de Leibniz, L'Ens. Math., 39 (1993), 269-293.

[6] Omirov. B. A., On the Derivations of Filiform Leibniz Algebras, Mathematical Notes, 5(2005), 677-685.

[7] Albeverio, S.; Ayupov, Sh. A.; Omirov, B. A., On nilpotent and simple Leibniz algebras, Comm. in Algebra 33(2005), 159-172.

[8] Ayupov, Sh. A.; Omirov, B. A., On Leibniz algebra, Algebra and Operator Theory. Proceeding of the Colloquium in Tashkent (1997), Kluwer (1998), 1-13.

[9] Ayupov, Sh. A.; Omirov, B. A., On 3-dimensional Leibniz algebra, Uzbek Math. (1999), 9-14.

[10] AL-hossain, A. A.; Khiyar, A. A., Derivations of some Filiform Leibniz algebras. pure and Applied mathematics Journal. Vol.3, No. 6, (2014), 121-125.

[11] Alnashri. A. A., Derivations of Second type of algebra of first class Filiform Leibniz algebras of Dimension Derivation $(n+1)$, International Journal of Advanced Scientific and Technical Research, Vol. 3, No. 5, (2015), 29-43.

[12] Alnashri. A. A., Derivations of one type of algebra of First class Filiform Leibniz algebras of Dimension Derivation $(n+1)$, International Journal of Advanced Scientific and Technical Research, Vol. 1, No. 5, (2015), 41-55. 